\title{
Autoimmun- erkrankungen
}

\section{Ein Risiko für sich \\ Systemische Sklerose lässt auch Koronarien verkalken}

Die systemische Sklerose ist ein unabhängiger Risikofaktor für Arteriosklerose. Um den Einfluss der Bindegewebskrankheit auf die Koronarverkalkung zu prüfen, verglichen Forscher aus Hong Kong 53 Patienten mit systemischer Sklerose und 106 Kontrollpersonen. Untersucht wurden der Koronararterien-Calcium-Score und kardiovaskuläre Risikofaktoren sowie bei den Patienten mit systemischer Sklerose die Krankheitsaktivität mithilfe des C-reaktiven Proteins (CRP) und dem Antiphospholipid-Antikörper. Dabei stellte sich heraus, dass 57\% der Patienten mit systemischer Sklerose mittel bis schwer verkalkte Herzkranzgefäße hatten. In der Kontrollgruppe war es nur 29\%. Zudem lagen der LDL- und - allerdings auch der HDL-Wert sowie der Blutdruck bei Patienten mit systemischer Sklerose deutlich unter den Werten der Kontrollgruppe. Mok MY et al, Arthritis Rheum 2011, 63:1387

\section{Basedow: Selen hilft dicken Augen}

> Patienten mit Morbus Basedow, die unter einer endokrinen Orbitopathie leiden, profitieren von einer Selen-Therapie. Das Spurenelement, das in verschiedenen Redox-Enzymen vorkommt, konnte in einer Studie mit 159 Patienten den ophthalmologischen Befund und die Lebensqualität signifikant verbessern. Zweimal täglich erhielten die Probanden $100 \mu \mathrm{g}$. Die Wirkung des ebenfalls untersuchten Pentoxifyllins, einem Phosphodiesterase-Inhibitor, dem antientzündliche und immunmodulierende Eigenschaften zugesprochen werden, unterschied sich hingegen nicht vom Placebo.

Marcocci C et al, N Engl J Med 2011, 364:1920

\section{Stress doch kein Risikofaktor für MS}

> Schwerer Stress sowie psychisch oder körperlich belastende Ereignisse lösen keine multiple Sklerose aus. Zwar haben Studien bereits gezeigt, dass Stress sich auf das Exazerbationsrisiko von MS-Patien-

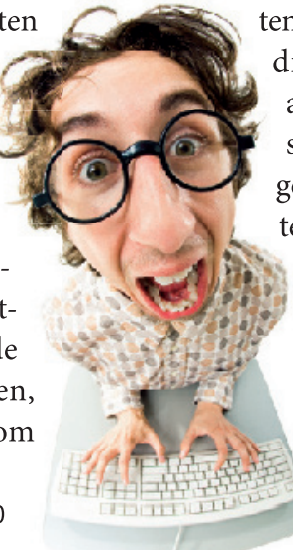
die Krankheitsentstehung an sich. So konnten Forscher der Universität Bergen anhand zweier Kohortenstudien mit 121.700 bzw.

116.671 Krankenschwestern weder bei beruflichem noch privatem Stress ein erhöhtes MS-Risiko feststellen. Riise T et al, Neurology 2011, 76:1866

Stress, aber kein MS! en auswirkt, jedoch nicht auf

Lupus erythematodes: Dem Herzinfarkt vorbeugen

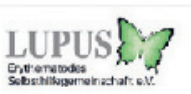

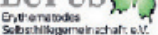

Lupus-Pass Notfall und Prävention
Mit einem Pass zur Prävention kardiovaskulärer Ereignisse sollen sich Patienten mit systemischem Lupus erythematodes künftig selbst über ihre Risiken aufklären. So erläutert der Lupus-Pass mittels Ampel-Schema, welche Lebensgewohnheiten sich gut (grün) und welche sich schlecht (rot) aufs Herz auswirken. www.lupus.rheumanet.org
Dr. Swanett Koops, stellvertretende Chefredakteurin

\section{Von Ziegen und dem „Horror autotoxicus"}

Mit einer Ziege fing alles an. Denn als Paul Ehrlich dem Tier im Rahmen seiner Forschungen Schafsblut injizierte, stellte er fest, dass die fremden Blutzellen gleich vernichtet wurden. Als er der Ziege ihr eigenes Blut verabreichte, passierte nichts. Das Immunsystem konnte offensichtlich zwischen "eigen" und "fremd" unterscheiden und griff seine eigenen Zellen nicht an. Diese Unterscheidung, von Ehrlich auch als „Horror autotoxicus" bezeichnet, ist lebensnotwendig und trotzdem nicht bei allen Menschen intakt. Lesen Sie in unserem Schwerpunkt zum Thema Autoimmunerkrankungen über die Therapie von Rheuma bei Kindern, wieso man Patienten mit Autoimmunerkrankungen impfen sollte und wie sich der Lupus erythematodes auf der Haut offenbart. Und auch die Allergie fehlt nicht. Zwar ist sie ja nicht im klassischen Sinn eine Autoimmunerkrankung, doch auch hier läuft das Immunsystem Amok. Eine informative Lektüre wünscht
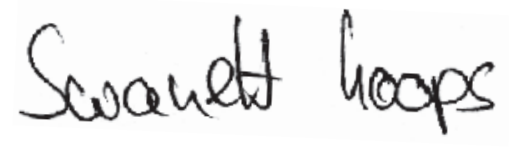

\section{springermedizin.de}

Top gelesen von

Hausärzten

Nicht jedes Bauchgrimmen ist EHEC Vorsicht nach Herzinfarkt - Lebensgefahr durch Schmerzmittel Was dürfen Epilepsiepatienten, was nicht?

Loggen Sie sich ein und nutzen Sie die Fülle von Beiträgen auf springermedizin.de! Die oben genannten Artikel finden Sie, in dem Sie die Schlagworte in die Suche eingeben. 